02

\title{
Электронное строение и спектры трифениламинов, функционализированных фенилэтинильными группами
}

\author{
(C) Г.В. Барышников ${ }^{1}$, В.А. Минаева ${ }^{1}$, Б.Ф. Минаев ${ }^{1}$, М. Григорас ${ }^{2}$ \\ ${ }^{1}$ Division of Theoretical Chemistry and Biology, School of Biotechnology, KTH Royal Institute of Technology, \\ 10691 Stockholm, Sweden \\ 2 „Petru Poni“ Institute of Macromolecular Chemistry, Electroactive Polymers Department, \\ 700487 lasi, Romania \\ e-mail: glibar@kth.se,minaeva@cdu.edu.ua, bfmin@rambler.ru
}

Поступила в редакцию 15.06.2017 г.

Рассмотрены особенности электронного строения, ИК и УФ видимые спектры серии трифениламинов, замещенных фенилэтинильными группами. Анализ выполнен на уровне теории функционала плотности и ее нестационарного варианта в сравнении с экспериментальными данными ИК и электронной спектроскопии. Показано, что в возбужденном состоянии происходит изменение альтернации одинарных, двойных и тройных связей в соответствии с характером связывания-разрыхления по низшей вакантной молекулярной орбитали. Найдено, что постепенное введение дополнительных фенилэтинильных групп не вызывает частотных сдвигов в ИК спектрах исследованных молекул, но заметно влияет на интенсивность соответствующих ИК полос. Аналогичный эффект наблюдается и в электронных спектрах поглощения данных соединений. Это может быть использовано для оптического тюнинга трифениламинов как перспективных материалов для органических светодиодов и солнечных батарей.

DOI: $10.21883 /$ OS.2018.01.45358.144-17

\section{Введение}

Звездообразные производные трифениламина на сегодняшний день привлекают значительное внимание исследователей в связи с перспективными оптическими свойствами этих соединений [1-5] и их высокой термои электростабильностью [6,7]. Свободный (нефункционализированный) трифениламин является оптически „прозрачным“ материалом и лишь слабо поглощает электромагнитное излучение в ближнем УФ диапазоне (в области 250-340 nm) [8]. Однако введение в состав трифениламинового основания ароматических сопряженных заместителей позволяет расширить диапазон поглощения трифениламинов в видимую область $[3,7,9-11]$. В случае если заместитель по своей природе электроно-акцепторный, удается также сильно усилить интенсивность поглощения света за счет возникновения электронных переходов с переносом заряда (ПЗ) от электроно-донорного трифениламинового ядра к электроно-акцепторным заместителям [9-11]. Рост интенсивности поглощения обусловлен возникновением большого дипольного момента перехода за счет неполного пространственного разделения молекулярных орбиталей (MO), ответственных за электронный переход. Очевидно, что усиление акцепторных свойств заместителей должно вызывать пространственную локализацию данных МО, что, в свою очередь, будет способствовать снижению интенсивности поглощения света и появлению „темновых“ сильно поляризованных электронных состояний цвиттерионного типа. Именно поэтому использование производных трифениламина в роли светопоглотителей или люминофоров (чаще флуорофоров) требует введения дополнительных $\pi$-связывающих (линкерных) групп между донорными и акцепторными фрагментами для обеспечения делокализации МО на этих фрагментах [3,7,9-11]. Такие соединения общепринято обозначать записью $\mathrm{D}-\pi-\mathrm{A}$, где $\mathrm{D}, \pi$ и $\mathrm{A}-$ донорный, линкерный и акцепторный фрагменты соответственно.

В настоящей работе нами рассмотрены особенности электронного строения, ИК и электронные спектры поглощения недавно синтезированных производных трифениламина (D), замещенных одной, двумя или тремя фенилэтинильными $\pi$-А-группами (рис. 1 ).

Данные соединения интенсивно поглощают свет на границе видимого и УФ диапазонов [10], а также демонстрируют размерно-зависимую флуоресценцию в области 400-450 nm, что позволяет их использовать в качестве сенсоров и активных сред в устройствах органической электроники (сенсибилизированные красителем солнечные батареи и органические светодио-
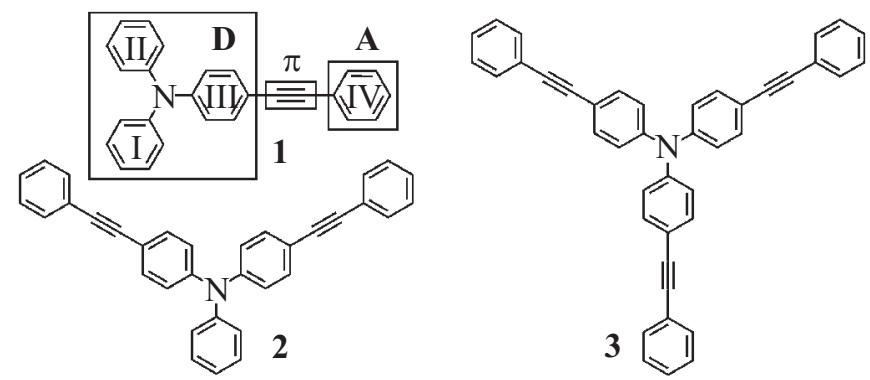

Рис. 1. Строение молекул $N, N$-дифенил-4-(фенилэтинил)анилина (1), $N$-фенил-4-(фенилэтинил)- $N$-(4-(фенилэтинил)фенил)анилина (2) и трис(4-(фенилэтинил)фенил)амина (3). 
ды) $[4,12,13]$. Сочетание квантово-химических методов исследования с данными ИК и электронной спектроскопии позволило нам проанализировать поведение молекул трифениламинов в возбужденном состоянии, а также соотнести наблюдаемые ИК полосы поглощения с конкретными типами колебаний, что является важным инструментом для анализа электронно-колебательных спектров изучаемых соединений.

\section{Объекты и методы}

Исследуемые молекулы $N, N$-дифенил-4-(фенилэтинил)анилина (1), $N$-фенил-4-(фенилэтинил)- $N$-(4-(фенилэтинил)фенил)анилина (2) и трис(4-(фенилэтинил)фенил)амина (3) были получены по реакции Соногаширы взаимодействием йод-замещенных трифениламинов с соответствующим количеством фенилацетилена по методике, описанной в работе [10]. Строение молекул доказано методом ЯМР. ${ }^{1} \mathrm{H}$ ЯМР спектры снимались при комнатной температуре на спектрометре BrukerAvance $\mathrm{DRX}-400$ в растворе $\mathrm{CDCl}_{3}$. ИК спектры исследуемых соединений были записаны на фурье-спектрометре DIGILAB-FTS 2000, используя образцы, прессованные c бромидом калия. Электронные спектры поглощения записаны с помощью спектрофотометра Specord 200 для $1 \cdot 10^{-5} \mathrm{M}$ растворов в хлороформе.

Квантово-химическая оптимизация равновесных геометрических параметров основного синглетного и возбужденного триплетного состояний молекул 1-3 выполнена методом DFT с использованием функционала B3LYP $[14,15]$ и базиса атомных орбиталей 6-31G(d) [16]. Этим же методом рассчитаны ИК спектры исследуемых молекул в основном синглетном состоянии. Все частоты колебаний оказались действительными, что указывает на нахождение истинного минимума на гиперповерхности полной энергии молекул. Рассчитанные частоты нормальных колебаний (НК) были скорректированы с использованием следующих масштабирующих множителей: 0.954 - для высокочастотной области и $0.970-$ для остальной части спектра. Данные значения позволяют достичь наилучшего согласия рассчитанных частот колебаний с экспериментально наблюдаемыми спектрами. Для расчета энергии и интенсивности синглетсинглетных электронных переходов нами использованы два гибридных обменно-корреляционных функционала B3LYP [14,15] и BMK [17] с разной степенью учета нелокального хартри-фоковского обмена (20 и $42 \%$ соответственно) в рамках зависимой от времени теории функционала плотности (TD DFT) [18] с использованием аналогичного базиса 6-31G(d) [16]. Для учета эффекта растворителя на энергию и интенсивность электронных переходов нами была использована модель поляризуемого континуума РСМ [19] (модельный растворитель хлороформ).

Рассчитанные спектры исследованных молекул построены с помощью программы SWizard [20] (полуши- рина линии равна $10 \mathrm{~cm}^{-1}$ для ИК спектров, функция распределения Лоренца, и $2500 \mathrm{~cm}^{-1}$ - для электронных спектров, функция распределения Гаусса). Определение форм нормальних колебаний проведено на основе анимации рассчитанных форм НК. Расчеты выполнены в рамках программного пакета Gaussian 09 (Revision D.01) [21].

\section{Результаты и обсуждение}

Молекула свободного основания трифениламина имеет характерную форму „пропеллера“ и соответствует пространственной группе симметрии $C_{3}$. Фенилэтинильные заместители в $n$-положениях размещаются в одной плоскости с фенильными группами трифениламина, образуя дифенилэтиновые „ветви“, обозначенные буквами А, В и С на рис. 2. В основном состоянии молекул 1-3 все дифенилэтиновые фрагменты идентичны и имеют строгую альтернацию связей С4-C7 (одинарная), С7-C8 (тройная) и С $8-\mathrm{C} 9$ (одинарная). При возбуждении в первое триплетное состояние наблюдается перераспределение длин этих связей таким образом, что образуется сильно сопряженный фрагмент C4C7C8C9, как это показано на рис. 2.

Связь $\mathrm{N}-\mathrm{C} 1$ заметно сокращается при возбуждении в триплет, а в бензольных кольцах наблюдается частичное перераспределение длин и порядков связей таким образом, что более длинные связи в основном

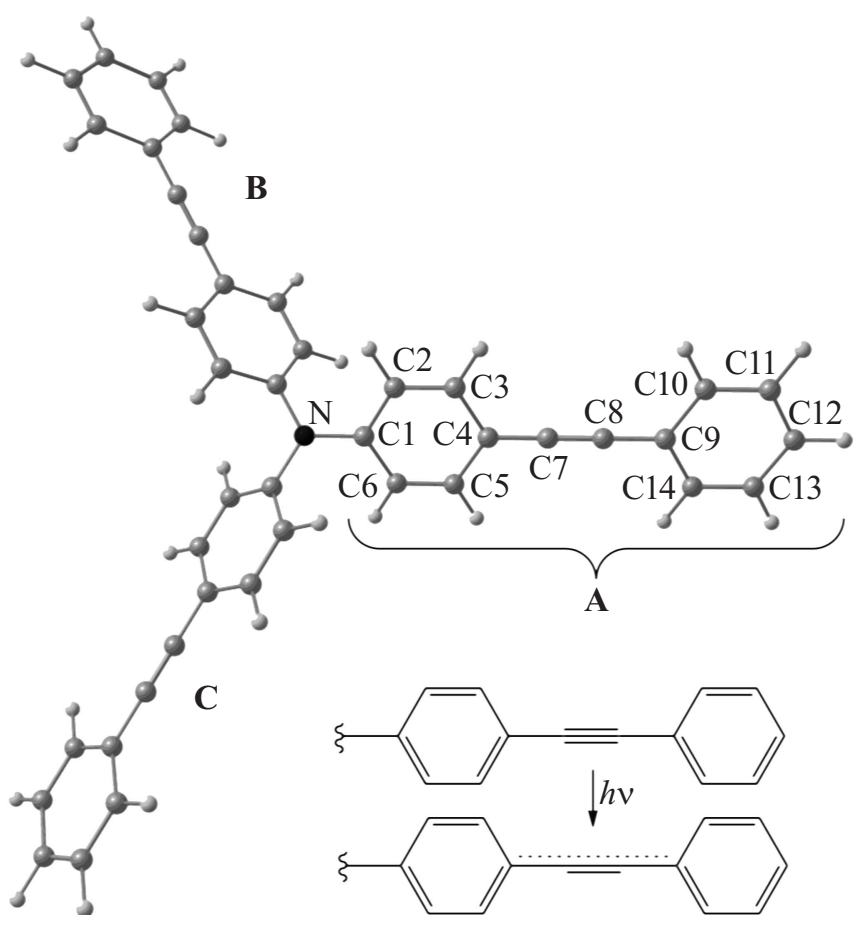

Рис. 2. Строение молекулы трис(4-(фенилэтинил)фенил)амина (3) с обозначением дифенилэтиновых фрагментов и нумерацией атомов в них. На вставке показано преобразование тройной связи при возбуждении в состояния $S_{1}$ и $T_{1}$. 
Таблица 1. Длины связей $(\AA)$, рассчитанные методом B3LYP/6-31G(d) для основного синглетного $\left(S_{0}\right)$ и первого возбужденного триплетного $\left(T_{1}\right)$ состояний молекул $1-3$

\begin{tabular}{c|c|c|c|c|c|c|c|c}
\hline Молекула & \multicolumn{2}{|c|}{1} & \multicolumn{2}{c|}{2} & & \multicolumn{2}{c}{3} \\
\hline Связь & $S_{0}(\mathrm{~A})$ & $T_{1}(\mathrm{~A})$ & $S_{0}(\mathrm{~A}, \mathrm{~B})$ & $T_{1}(\mathrm{~A})$ & $T_{1}(\mathrm{~B})$ & $S_{0}(\mathrm{~A}, \mathrm{~B}, \mathrm{C})$ & $T_{1}(\mathrm{~A})$ & $T_{1}(\mathrm{~B}, \mathrm{C})$ \\
\hline $\mathrm{N}-\mathrm{C} 1$ & 1.420 & 1.402 & 1.414 & 1.400 & 1.414 & 1.420 & 1.400 & 1.418 \\
$\mathrm{C} 1-\mathrm{C} 2$ & 1.406 & 1.436 & 1.409 & 1.436 & 1.410 & 1.406 & 1.437 & 1.408 \\
$\mathrm{C} 1-\mathrm{C} 6$ & 1.406 & 1.436 & 1.409 & 1.436 & 1.410 & 1.406 & 1.437 & 1.408 \\
$\mathrm{C} 2-\mathrm{C} 3$ & 1.388 & 1.362 & 1.385 & 1.362 & 1.385 & 1.388 & 1.361 & 1.386 \\
$\mathrm{C} 5-\mathrm{C} 6$ & 1.388 & 1.362 & 1.385 & 1.362 & 1.385 & 1.388 & 1.361 & 1.386 \\
$\mathrm{C} 3-\mathrm{C} 4$ & 1.410 & 1.462 & 1.413 & 1.461 & 1.413 & 1.410 & 1.460 & 1.412 \\
$\mathrm{C} 4-\mathrm{C} 5$ & 1.410 & 1.462 & 1.413 & 1.461 & 1.413 & 1.410 & 1.460 & 1.412 \\
$\mathrm{C} 4-\mathrm{C} 7$ & 1.422 & 1.359 & 1.419 & 1.360 & 1.419 & 1.422 & 1.362 & 1.419 \\
$\mathrm{C} 7-\mathrm{C} 8$ & 1.217 & 1.252 & 1.218 & 1.251 & 1.218 & 1.217 & 1.249 & 1.218 \\
$\mathrm{C} 8-\mathrm{C} 9$ & 1.424 & 1.383 & 1.423 & 1.385 & 1.423 & 1.424 & 1.387 & 1.423 \\
C9-C10 & 1.410 & 1.433 & 1.410 & 1.432 & 1.410 & 1.410 & 1.431 & 1.410 \\
$\mathrm{C} 9-\mathrm{C} 14$ & 1.410 & 1.433 & 1.410 & 1.432 & 1.410 & 1.410 & 1.431 & 1.410 \\
$\mathrm{C} 10-\mathrm{C} 11$ & 1.392 & 1.383 & 1.392 & 1.384 & 1.392 & 1.392 & 1.384 & 1.392 \\
$\mathrm{C} 13-\mathrm{C} 14$ & 1.392 & 1.383 & 1.392 & 1.384 & 1.392 & 1.392 & 1.384 & 1.392 \\
$\mathrm{C} 11-\mathrm{C} 12$ & 1.397 & 1.406 & 1.397 & 1.405 & 1.397 & 1.397 & 1.404 & 1.397
\end{tabular}

состоянии $S_{0}(\mathrm{C} 1-\mathrm{C} 2$ и $\mathrm{C} 1-\mathrm{C} 6, \mathrm{C} 3-\mathrm{C} 4$ и $\mathrm{C} 4-\mathrm{C} 5$, C9-C10 и $\mathrm{C} 9-\mathrm{C} 14, \mathrm{C} 11-\mathrm{C} 12$ и $\mathrm{C} 12-\mathrm{C} 13)$ становятся еще более длинными, а короткие $(\mathrm{C} 2-\mathrm{C} 3$ и $\mathrm{C} 5-\mathrm{C} 6$, C10-C11 и $\mathrm{C} 13-\mathrm{C} 14)$ дополнительно сокращаются. Данные изменения связаны с тем, что строение молекул $1-3$ в состоянии $T_{1}$ в значительной мере определяется структурой низшей вакантной молекулярной орбитали (LUMO), на которую переходит электрон с дважды занятой высшей занятой молекулярной орбитали (НОMO) при возбуждении в триплет. Первое синглетное возбужденное состояние $\left(S_{1}\right)$ имеет аналогичную триплету HOMO-LUMO-конфигурацию, что указывает на аналогичный характер связывания-разрыхления, т.е. строение молекул $1-3$ в состоянии $S_{1}$ можно предположить аналогичным их строению в состоянии $T_{1}$, оптимизация которого была выполнена на уровне неограниченного по спину формализма Кона-Шема (UB3LYP/6-31G(d)). Важно отметить, что для всех трех молекул возбуждение в состояние $T_{1}$ вызывает структурные изменения лишь в одной из дифенилэтиновых ветвей А, в то время как фрагменты В и С почти не претерпевают изменений (табл. 1). Это является следствием локализации возбуждения на одной из „ветвей“ звездообразных молекул (в данном случае фрагмент А) вследствие наличия ПЗ-характера состояний $S_{1}$ и $T_{1}$ [4]. Как следствие, молекулы 1-3 в возбужденном состоянии приобретают биполярную структуру, т.е. становятся сильно поляризованными, о чем свидетельствует сильный рост дипольного момента (в среднем на $3 \mathrm{D}$ ) в состоянии $T_{1}$ относительно основного состояния $S_{0}(1.1 \mathrm{D} v s 4.0 \mathrm{D}$, $0.8 \mathrm{D} v s 3.5 \mathrm{D}, 0.0 \mathrm{D} v s 3.3 \mathrm{D}$ для молекул 1,2 и 3 соответственно). Это, в свою очередь, обусловливает положительную сольватохромию молекул $1-3$, зависящую от полярности растворителя. Данное свойство является очень важным для оптической настройки поглощения света и флуоресценции молекул $1-3$, что может быть использовано для перестройки цвета соответствующих солнечных элементов и органических светодиодов, подобно тому, как это предлагается в работах $[3,4]$ для родственных звездообразных систем.

\section{Электронные спектры поглощения}

Рассчитанные электронные спектры молекул 1-3 содержат две основные полосы поглощения - интенсивную длинноволновую в области $300-400 \mathrm{~nm}$ и менее интенсивную коротковолновую в области $250-300 \mathrm{~nm}$ (рис. 3, табл. 2). В соответствии с результатами TD DFT расчетов и построения изоповерхностей соответствующих $\mathrm{MO}$, можно сказать, что первая (длинноволновая) полоса поглощения обусловлена синглет-синглетными электронными переходами $\pi \pi^{*}$-типа с ПЗ от трифениламинового „донора“ к б боковым фенилэтинильным ответвлениям (рис. 4). Несмотря на ПЗ-природу, данные переходы имеют очень большую интенсивность, что обусловлено неполным пространственным разделением MO, между которыми осуществляется электронный переход. Фактически имеются обширные области (тройная связь и прилегающая $N$-фенильная группа, рис. 4), на которых эти МО имеют ненулевые коэффициенты разложения определенного знака, благодаря чему формируются большие величины дипольного момента перехода [4,22-24].

Важно отметить, что в экспериментальных спектрах молекул 1 и 2 наблюдается ярко выраженная колебательная структура (хорошо видны полосы 0-1, 

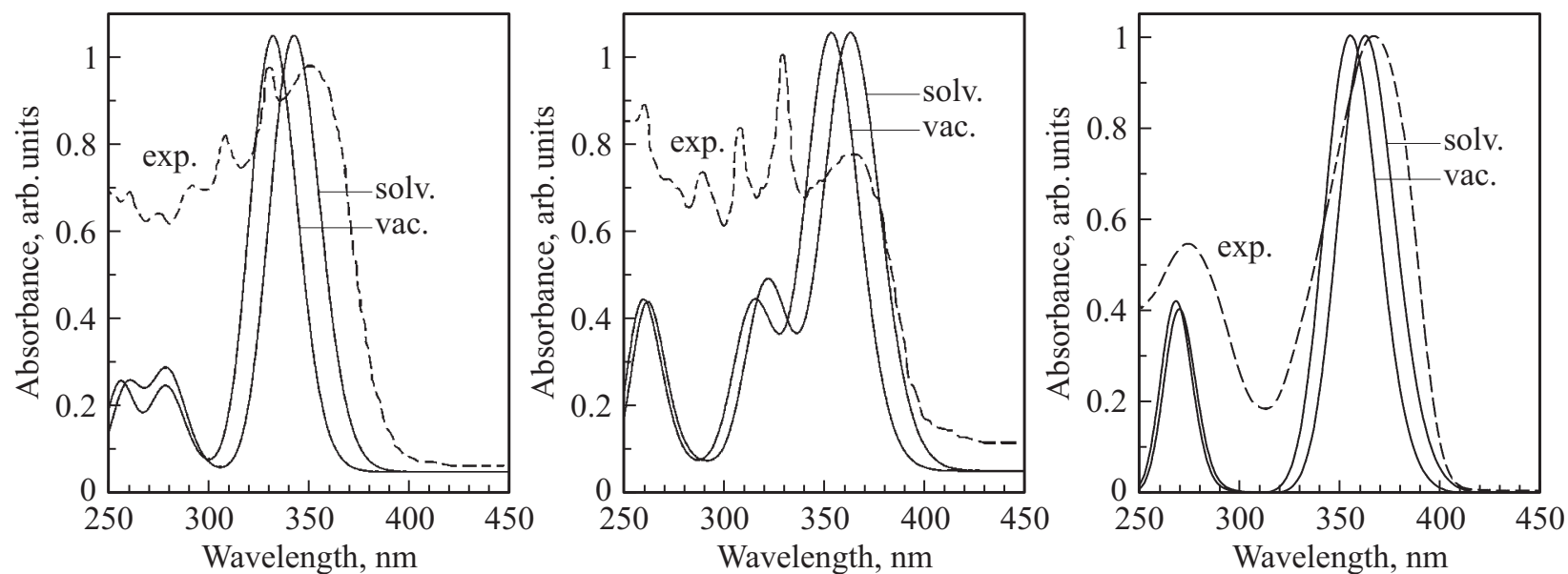

Рис. 3. Электронные спектры поглощения молекул 1 (слева), 2 (по центру) и 3 (справа), измеренные экспериментально в растворах хлороформа (штриховые линии „ехр“), а также рассчитанные методом TD DFT/BMK/6-31G(d) в вакуумном (vac) и PCM (solv) приближениях.

0-2 и 0-3 с монотонно убывающей интенсивностью, рис. 3), которая, очевидно, принадлежит прогрессии валентного колебания тройной связи $-\mathrm{C} \equiv \mathrm{C}-$ в первом возбужденном синглетном состоянии. Мы моделируем его структуру через оптимизацию триплетного состояния $T_{1}$, что легче и точнее достигается в рамках неограниченного по спину метода UB3LYP. B основном состоянии $S_{0}$ это колебание дает в ИК спектре полосу низкой интенсивности в области $2200 \mathrm{~cm}^{-1}$, но в возбужденном триплетном состоянии эта полоса смещается в область $1800 \mathrm{~cm}^{-1}$, что хорошо согласуется с экспери- ментальной оценкой интервалов полосы $S_{0}-S_{1}$ между электронно-колебательными сателлитами $\approx 1850 \mathrm{~cm}^{-1}$. В спектре молекулы 2 полоса 0-1 совпадает с электронным переходом в состояние $S_{2}$ (рис. 3), который, очевидно, усиливает ее, чего не наблюдается в спектре молекулы 1 , для которой состояние $S_{2}(288 \mathrm{~nm})$ лежит намного выше полосы 0-1 (331 nm) и имеет очень малую интенсивность $(f=0.002$, не показано в табл. 1). В электронном спектре молекулы 3 колебательная структура не наблюдается вовсе (рис. 3), что, очевидно, обусловлено высокой симметрией молекулы,

Таблица 2. Рассчитанные и экспериментальные спектральные характеристики молекул 1-3: длины волн электронных переходов $(\lambda, \mathrm{nm})$, силы осциллятора $(f)$ и отнесение электронных переходов для расчетов в вакууме (vac) и в растворителе в PCM приближении (solv - хлороформ), проведенные методами TD DFT/B3LYP/6-31G(d) и TD DFT/BMK/6-31G(d)

\begin{tabular}{|c|c|c|c|c|c|c|}
\hline$S_{n}$ & $\lambda_{\mathrm{B} 3 \mathrm{LYP}}^{\mathrm{vac}} / \lambda_{\mathrm{B} 3 \mathrm{LYP}}^{\mathrm{solv}}$ & $\lambda_{\mathrm{BMK}}^{\mathrm{vac}} / \lambda_{\mathrm{BMK}}^{\mathrm{solv}}$ & $\lambda_{\exp }$ & $f_{\mathrm{B} 3 \mathrm{LYP}}^{\mathrm{vac}} / f_{\mathrm{B} 3 \mathrm{LYP}}^{\mathrm{solv}}$ & $f_{\mathrm{BMK}}^{\mathrm{vac}} / f_{\mathrm{BMK}}^{\mathrm{solv}}$ & Отнесение \\
\hline \multicolumn{7}{|c|}{1} \\
\hline $\begin{array}{l}S_{1} \\
S_{3} \\
S_{4}\end{array}$ & $\begin{array}{l}370 / 384 \\
310 / 309 \\
286 / 288\end{array}$ & $\begin{array}{l}332 / \mathbf{3 4 3} \\
279 / \mathbf{2 8 0} \\
257 / \mathbf{2 6 1}\end{array}$ & $\begin{array}{l}352 \\
276 \\
260\end{array}$ & $\begin{array}{l}1.02 / 1.15 \\
0.18 / 0.24 \\
0.36 / 0.43\end{array}$ & $\begin{array}{l}1.22 / \mathbf{1 . 3 7} \\
0.23 / \mathbf{0 . 3 0} \\
0.22 / \mathbf{0 . 2 4}\end{array}$ & $\begin{array}{l}91-92 \\
91-94 \\
90-92\end{array}$ \\
\hline \multicolumn{7}{|c|}{2} \\
\hline $\begin{array}{l}S_{1} \\
S_{2} \\
S_{4} \\
S_{6}\end{array}$ & $\begin{array}{l}400 / 412 \\
360 / 367 \\
306 / 310 \\
291 / 292\end{array}$ & $\begin{array}{l}354 / \mathbf{3 6 3} \\
315 / \mathbf{3 2 2} \\
269 / \mathbf{2 7 2} \\
259 / \mathbf{2 6 2}\end{array}$ & $\begin{array}{l}366 \\
329 \\
261\end{array}$ & $\begin{array}{l}1.31 / 1.46 \\
0.34 / 0.47 \\
0.40 / 0.41 \\
0.53 / 0.65\end{array}$ & $\begin{array}{l}1.59 / \mathbf{1 . 7 4} \\
0.62 / \mathbf{0 . 7 6} \\
0.17 / \mathbf{0 . 1 4} \\
0.51 / \mathbf{0 . 5 6}\end{array}$ & $\begin{array}{l}117-118 \\
117-119 \\
116-118 \\
116-119\end{array}$ \\
\hline \multicolumn{7}{|c|}{3} \\
\hline$S_{1,2}$ & $407 / 416$ & $355 / 363$ & 369 & $1.09 / 1.26$ & $1.45 / 1.62$ & $\begin{array}{l}143-144\left(S_{1}\right) \\
143-145\left(S_{2}\right)\end{array}$ \\
\hline$S_{5,6}$ & $312 / 313$ & $269 / 271$ & 275 & $0.66 / 0.70$ & $0.60 / 0.65$ & $\begin{array}{c}{[141-144]+[142-145]} \\
\left(S_{5}\right) \\
{[142-144]-[141-145]} \\
\left(S_{6}\right)\end{array}$ \\
\hline
\end{tabular}




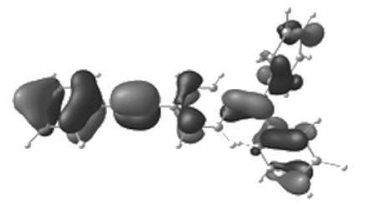

HOMO - 1 (90)

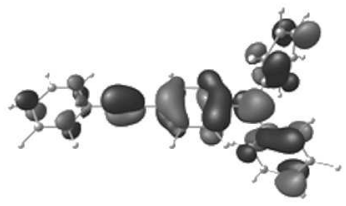

HOMO (91)

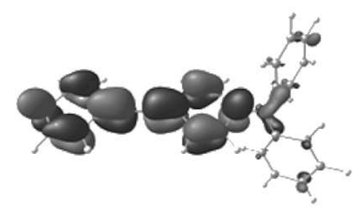

LUMO (92)

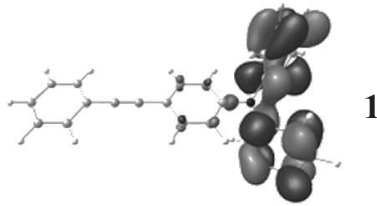

LUMO + 2 (94)
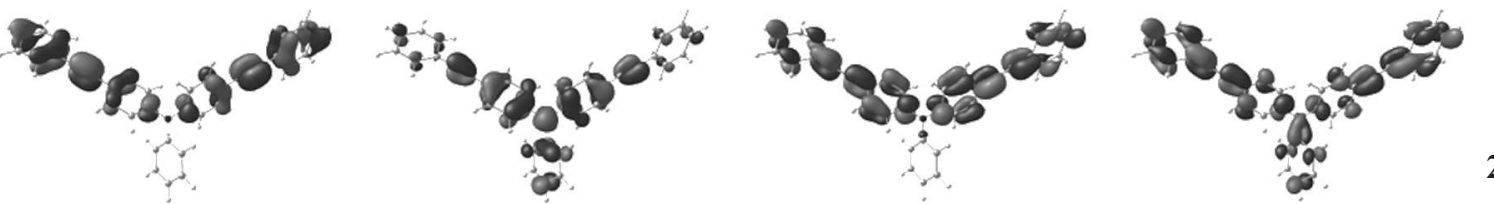

HOMO - 1 (116)

HOMO (117)

LUMO (118)

LUMO + 1 (119)

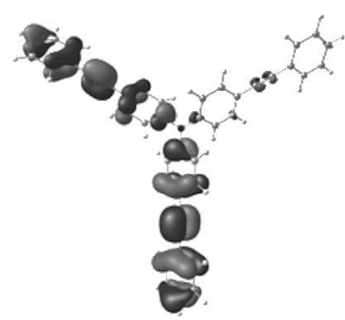

HOMO - 1 (142)

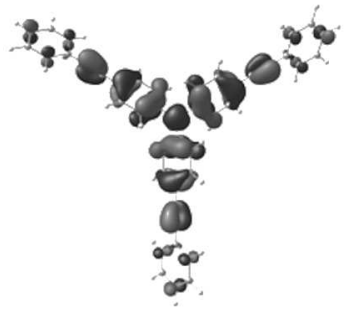

HOMO (143)

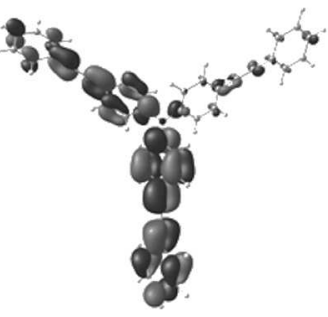

LUMO (144)

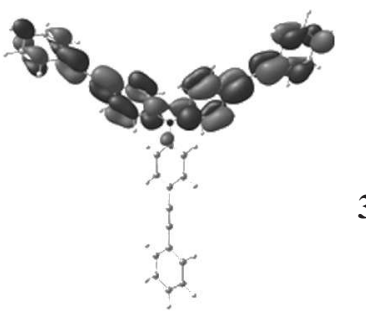

LUMO + 1 (145)

\section{2}

3

Рис. 4. Форма граничных молекулярных орбиталей молекул 1-3, рассчитанная методом B3LYP/6-31G(d) (контролирующее значение изоконтура 0.025 a.u.)
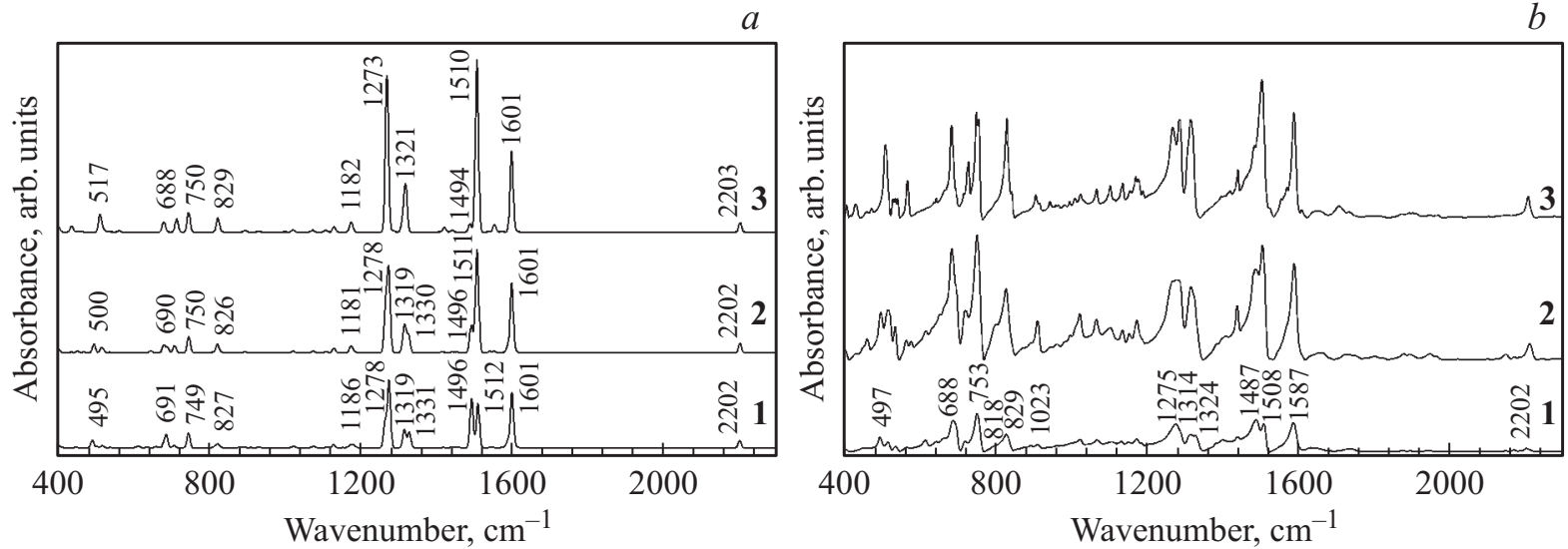

Рис. 5. Рассчитанные методом В3LYP/6-31G(d) (a) и экспериментально измеренные $(b)$ ИК спектры поглощения молекул $1-3$ для спектральной области $2400-400 \mathrm{~cm}^{-1}$.

налагающей запрет на соответствующие электронноколебательные переходы.

Как можно видеть из табл. 2, энергия и интенсивность электронных переходов в спектрах соединений 1-3 сильно зависит от выбора обменно-корреляционного функционала и в меньшей мере от растворителя. Зависимость от функционала является хорошо известной и обусловлена необходимостью повышенного учета нелокального хартри-фоковского обмена для ПЗ-состояний с неполным пространственным разделением соответствующих МО [25]. Слабое влияние растворителя на спектры поглощения обусловлено в первую очередь малой поляризацией молекул в основном состоянии, однако в состоянии $S_{1}$ с примесью ПЗ эффект растворителя проявляется сильнее. Это приводит к малому батохромному сдвигу под влиянием растворителя, явно улучшающему согласие с опытом (рис. 3). Можно предсказать, что П3-природа возбужденного состояния $S_{1}$ должна давать 
Таблица 3. Данные по ИК спектру $N, N$-дифенил-4-(фенилэтинил)анилина (1)

\begin{tabular}{|c|c|c|c|c|}
\hline $\begin{array}{c}\text { № } \\
\text { моды }\end{array}$ & $\begin{array}{l}v_{\text {pacq }} \\
\mathrm{cm}^{-1}\end{array}$ & $\begin{array}{c}I, \\
\mathrm{~km} / \text { mole }\end{array}$ & $\begin{array}{l}v_{\text {эксп }} \\
\mathrm{cm}^{-1}\end{array}$ & Тип колебания* \\
\hline 113 & 2202 & 43.1 & 2202 & $v(-\mathrm{C} \equiv \mathrm{C}-)$ \\
\hline 109 & 1601 & 231.4 & 1587 & $v_{\mathrm{s}}(\mathrm{C}=\mathrm{C}), \mathrm{I}-\mathrm{IV}$ \\
\hline 104 & 1512 & 255.8 & 1508 & $v_{\text {as }}(\mathrm{C}=\mathrm{C}), \mathrm{III}, \mathrm{IV}$ \\
\hline 103 & 1496 & 141.0 & \multirow{2}{*}{1487} & $v_{\text {as }}(\mathrm{C}=\mathrm{C}), \mathrm{I}, \mathrm{II}, v(\mathrm{C}-\mathrm{N})$, в фазе \\
\hline 102 & 1495 & 138.4 & & $v_{\text {as }}(\mathrm{C}=\mathrm{C}), \mathrm{I}, \mathrm{II}, v(\mathrm{C}-\mathrm{N})$, прф. \\
\hline 96 & 1331 & 82.1 & 1324 & $v(\mathrm{C}(\mathrm{III})-\mathrm{N}), v(\mathrm{C}-\mathrm{C})$, прф. \\
\hline 93 & 1319 & 57.0 & 1314 & $\begin{array}{c}v_{\text {as }}(\mathrm{C}=\mathrm{C}) \text { Кекуле, I, II, } v(\mathrm{C}-\mathrm{N}) \\
v(\mathrm{C}-\mathrm{C}), \text { прф. }\end{array}$ \\
\hline 88 & 1278 & 374.8 & \multirow{2}{*}{1275} & $v(\mathrm{C}(\mathrm{III})-\mathrm{N}), \delta(\mathrm{CH}), \mathrm{I}-\mathrm{III}$ \\
\hline 86 & 1267 & 144.5 & & $v(\mathrm{C}(\mathrm{I})-\mathrm{N})$ и $v(\mathrm{C}(\mathrm{II})-\mathrm{N})$, прф. \\
\hline 85 & 1186 & 14.3 & 1184 & $\delta(\mathrm{CH}), \mathrm{I}-\mathrm{III}, v(\mathrm{C}-\mathrm{N})$, в фазе \\
\hline 50 & 827 & 18.1 & 829 & $\gamma(\mathrm{CH})$, в фазе, III, $\gamma(\mathrm{CH})$, прф., II \\
\hline 48 & 820 & 11.4 & $\begin{array}{c}818 \\
\text { (плечо) }\end{array}$ & $\gamma(\mathrm{CH})$, в фазе, III, $\gamma(\mathrm{CH})$, прф., I, II \\
\hline 45 & 749 & 72.3 & 753 & $\gamma(\mathrm{CH})$, в фазе, I, II, IV \\
\hline 41 & 691 & 52.9 & 688 & Неплоские деф. бенз. колец, I, II \\
\hline 27 & 495 & 43.6 & 497 & Неплоские деф. бенз. колец, I, II \\
\hline
\end{tabular}

* Примечание. I, II, III, IV - нумерация бензольных колец (рис. 1); v - частота (волновое число), а также $v-$ валентное колебание; $I$ - интенсивность ИК поглощения; $\delta$ - деформационное колебание (изменение валентного угла); $\gamma$ - неплоское колебание; деф. — деформация; прф. - в противофазе; эксп - экспериментальный; расч - расчетный; s - симметричное колебание; as — асимметричное колебание.

более сильную сольватохромию в спектрах флуоресценции, наблюдаемую экспериментально для родственных соединений $[3,4]$.

В завершении следует отметить, что наиболее важным свойством изучаемых молекул 1-3 является очень высокая интенсивность синглет-синглетного первого электронного перехода, который обусловливает интенсивное поглощение квантов энергии на границе УФ и видимого диапазонов и последующую синюю флуоресценцию в области $410 \mathrm{~nm}$. Это открывает возможности для внедрения изучаемых молекул в структуру синих и белых органических светодиодов, так как именно синие флуорофоры являются наименее распространенными материалами для OLED устройств.

\section{ИК спектры}

В данном разделе нами дана интерпретация основных полос поглощения в ИК спектрах молекул $1-3$, а также обсуждается зависимость интенсивности ИК полос от количества фенилэтинильных групп, сопряженных с трифениламиновым ядром. В связи с этим интенсивности ИК полос были нормированы на единый максимум поглощения в каждой серии из трех экспериментальных и трех теоретически рассчитанных спектров (рис. 5, $a$ и $5, b$ соответственно).

Отнесение полос поглощения в экспериментальных ИК спектрах показано на рис. 5, $b$, в то время как на рис. 5, a сопоставлены теоретически рассчитанные кривые с указанием частот основных максимумов. Анализ ИК спектров показал, что с ростом числа фенилэтинильних групп интенсивность полос поглощения увеличивается как в результате увеличения количества групп $-\mathrm{C} \equiv \mathrm{C}-$ и образования вырожденных колебательных мод (без смешивания с другими типами колебаний) [26], так и вследствие возрастания дипольного момента колебаний с увеличением длины плеча при введении фенилэтинильной группы. Однако частотный сдвиг полос поглощения при этом не наблюдается, поэтому нами детально рассмотрен лишь ИК спектр молекулы 1, в то время как остальные рассчитанные спектры могут быть отнесены по аналогии со спектром этой молекулы.

Сильные полосы поглощения с максимумами 1587 и $1508 \mathrm{~cm}^{-1}$ в экспериментальном спектре соединения 1 (рис. 5, $b$, кривая 1) принадлежат соответственно симметричным и асимметричным колебаниям связей $\mathrm{C}=\mathrm{C}$ бензольных колец (расч.: 1601 и $1512 \mathrm{~cm}^{-1}$ соответственно, табл. 3 , рис. 5, $a, b$, кривая 1$)$. 
Поглощение при $1487 \mathrm{~cm}^{-1}$ (расч.: $1496 \mathrm{~cm}^{-1}$ ) вызвано участием связей $\mathrm{C}-\mathrm{N}$ в асимметричных скелетных колебаниях бензольных колец трифениламинового фрагмента. Дублетная полоса с максимумами 1324 и $1314 \mathrm{~cm}^{-1}$ (рис. 5, $b$, кривая 1 ) принадлежит соответственно асимметричным колебаниям аминогруппы (расч.: $1331 \mathrm{~cm}^{-1}$, табл. $\left.3, \nu_{96}\right)$ и колебаниям $v_{\mathrm{as}}(\mathrm{C}=\mathrm{C}$ ) Кекуле, смешанным со скелетными колебаниями аминогруппы и связей С-C фенилэтинильного фрагмента (расч.: $1319 \mathrm{~cm}^{-1}$, табл. 3, v93). Колебания связей $\mathrm{C}-\mathrm{N}$ образуют также сильную полосу с максимумом $1275 \mathrm{~cm}^{-1}$ и слабую полосу при $1184 \mathrm{~cm}^{-1}$ (расч.: 1278 и $1186 \mathrm{~cm}^{-1}$ ). Слабые полосы в области $1173-1067 \mathrm{~cm}^{-1}$ обусловлены деформационными колебаниями СН бензольных колец $(\delta \mathrm{CH})$. Неплоские деформации СН бензольных фрагментов $(\gamma \mathrm{CH})$ дают более интенсивные полосы (расч.: 827 и $749 \mathrm{~cm}^{-1}$, эксп.: 829 и $753 \mathrm{~cm}^{-1}$ ), но рассчитанные НК образуют не столь интенсивные полосы по сравнению с экспериментальными спектрами (рис. 5). Неплоские деформации самих скелетов бензольных колец имеют большую ИК интенсивность по сравнению с плоскими деформациями (эксп.: $1023 \mathrm{~cm}^{-1}$, расч.: $1026 \mathrm{~cm}^{-1}$ ) и дают в экспериментальном спектре слабую полосу поглощения при $497 \mathrm{~cm}^{-1}$ и сильную полосу при $688 \mathrm{~cm}^{-1}$. Поглощение тройной связи $-\mathrm{C} \equiv \mathrm{C}-$, рассчитанное нами при $2202 \mathrm{~cm}^{-1}$, достаточно интенсивное для наблюдения в ИК спектре (эксп.: $2202 \mathrm{~cm}^{-1}$ ). Напомним, что колебание тройной связи $-\mathrm{C} \equiv \mathrm{C}-$ в возбужденном синглетном состоянии молекул 1 и 2 дает отчетливую колебательную прогрессию, которая в силу особенностей симметрии отсутствует в спектрах поглощения и флуоресценции молекулы 3.

\section{Выводы}

1.Исследованные в работе трифениламины, функционализированные одной, двумя и тремя фенилэтинильными группами соответственно, являются типичными представителями молекул так называемых D-л-A-систем, в которых донорный трифениламиновый фрагмент (D) coпряжен с фенильной группой (A) посредством тройной $-\mathrm{C} \equiv \mathrm{C}-$ связи $(\pi)$.

2. Вследствие неполного пространственного разделения граничных МО, между которыми осуществляется возбуждение в первый синглет, дипольный момент этого перехода достаточно велик за счет ненулевой области наложения соответствующих волновых функций МО на общем $N$-фенилэтинильном фрагменте. Как следствие, изученные соединения интенсивно поглощают на границе ближнего УФ и видимого диапазонов, а также дают отчетливую флуоресценцию в синем диапазоне, зависящую от полярности растворителя.

3. В силу особенности электронного строения спектры поглощения молекул с одним и двумя фенилэтинильными заместителями имеют ярко выраженную колебательную структуру, обусловленную прогрессией трой- ной связи $-\mathrm{C} \equiv \mathrm{C}-$. В то же время трис (4-(фенилетинил)фенил)амин с тремя фенилэтинильными группами имеет предельно простой электронный спектр поглощения без какой-либо колебательной структуры в силу высокой симметрии молекулы.

4. Теоретически рассчитанные и экспериментально измеренные ИК спектры поглощения изученных систем хорошо соотносятся между собой по положению и интенсивности основных ИК полос. Кроме того, наблюдается пропорциональный рост интенсивности колебаний связи $-\mathrm{C} \equiv \mathrm{C}-$ и бензольных колец при постепенном введении фенилэтинильных групп в трифениламиновое ядро, что вызвано в основном складыванием интенсивностей однотипных колебательных мод.

Настоящая работа выполнена при поддержке Министерства образования и науки Украины (проект № 0115U000637), гранта Президента Украины для молодых ученых (проект№ 0117U001979), а также шведского фонда имени Карла Триггера (грант № CTS 16 : 536). Bce DFT расчеты проведены на базе Высокопроизводительного Вычислительного центра „Север“ (НPC2N) Шведского центра научных и параллельных исследований (SNIC) в рамках проекта „Мультифизическое моделирование молекулярных материалов“ (№ 020/11-23).

\section{Список литературы}

[1] Kreger K., Bäte M., Neuber C., Schmidt H.-W., Strohriegl P. // Adv. Funct. Mater. 2007. V. 17. N 17. P. 3456.

[2] Jin R. // J. Mol. Model. 2015. V. 21. P. 219.

[3] Matulaitis T., Kostiv N., Grazulevicius J.V., Peciulyte L., Simokaitiene J., Jankauskas V., Luszczynska B., Ulanski J. // Dyes Pigm. 2016. V. 127. P. 45.

[4] Baryshnikov G.V., Bondarchuk S.V., Minaeva V.A., Agren H., Minaev B.F. // J. Mol. Model. 2017. V. 23. P. 55.

[5] Min J., Luponosov Y.N., Ameri T., Elschner A., Peregudova S.M., Baran D., Heumüller T., Li N., Machui F., Ponomarenko S., Brabec C.J. // Organ. Electron. 2013. V. 14. N 1. P. 219.

[6] Zou Y., He Z., Zhao B., Liu Y., Yang C., Wu H., Cao Y. // Sci. Rep. 2015. V. 5. P. 17329.

[7] Shang H., Fan H., Liu Y., Hu W., Li Y., Zhan X. // Adv. Mater. 2011. V. 23. N 13. P. 1554.

[8] Schroeder W.A., Wilcox P.E., Trueblood K.N., Dekker A.O. // Anal. Chem. 1951. V. 23. N 12. P. 1740.

[9] Grigoras M., Catargiu A.M., Ivan T., Vacareanu L., Minaev B., Stromylo E. // Dyes Pigm. 2015. V. 113. P. 227.

[10] Grigoras M., Ivan T., Vacareanu L., Catargiu A.M., Tigoianu R. // J. Luminesc. 2014. V. 153. P. 5.

[11] Li W., Li Q., Duan C., Liu S., Ying L., Huang F., Cao Y. // Dyes Pigm. 2015. V. 113. P. 1.

[12] Minaev B., Baryshnikov G., Agren H. // Phys. Chem. Chem. Phys. 2014. V. 16. N 5. P. 1719.

[13] Baryshnikov G., Minaev B., Agren H. // Chem. Rev. 2017. V. 117. N 9. P. 6500.

[14] Becke A.D. // J. Chem. Phys. 1993. V. 98. N 7. P. 5648.

[15] Lee C., Yang W., Parr R.G. // Phys. Rev. B. 1988. V. 37. N 2. P. 785. 
[16] Francl M.M., Pietro W.J., Hehre W.J., Binkley J.S., DeFrees D.J., Pople J.A., Gordon M.S. // J. Chem. Phys. 1982. V. 77. N 7. P. 3654.

[17] Boese A.D., Martin J.M.L. // J. Chem. Phys. 2004. V. 121. N 8. P. 3405.

[18] Runge E., Gross E.K.U. // Phys. Rev. Lett. 1984. V. 52. N 12. P. 997.

[19] Miertuš S., Scrocco E., Tomasi J. // Chem. Phys. 1981. V. 55. N 1. P. 117.

[20] Gorelsky S.I. SWizard program, http://www.sg-chem.net/, University of Ottawa, Ottawa, Canada, 2013.

[21] Frisch M.J., Trucks G.W., Schlegel H.B., Scuseria G.E., Robb M.A., Cheeseman J.R., Scalmani G., Barone V., Mennucci B., Petersson G.A., Nakatsuji H., Caricato M., Li X., Hratchian H.P., Izmaylov A.F., Bloino J., Zheng G., Sonnenberg J.L., Hada M., Ehara M., Toyota K., Fukuda R., Hasegawa J., Ishida M., Nakajima T., Honda Y., Kitao O., Nakai H., Vreven T., Montgomery J.A., Jr., Peralta J.E., Ogliaro F., Bearpark M., Heyd J.J., Brothers E., Kudin K.N., Staroverov V.N., Kobayashi R., Normand J., Raghavachari K., Rendell A., Burant J.C., Iyengar S.S., Tomasi J., Cossi M., Rega N., Millam M.J., Klene M., Knox J.E., Cross J.B., Bakken V., Adamo C., Jaramillo J., Gomperts R., Stratmann R.E., Yazyev O., Austin A.J., Cammi R., Pomelli C., Ochterski J.W., Martin R.L., Morokuma K., Zakrzewski V.G., Voth G.A., Salvador P., Dannenberg J.J., Dapprich S., Daniels A.D., Farkas Ö., Foresman J.B., Ortiz J.V., Cioslowski J., Fox D.J. Gaussian 09, Revision D.01, Gaussian, Inc., Wallingford, CT, 2009.

[22] Minaev B.F., Baryshnikov G.V., Minaeva V.A. // Dyes Pigm. 2012. V. 92. N 1. P. 531.

[23] Барышников Г.В., Минаев Б.Ф., Минаева В.А. // Опт. и спектр. 2010. Т. 108. № 1. С. 18; Baryshnikov G.V., Minaev B.F., Minaeva V.A. // Opt. Spectrosc. 2010. V. 108. N 1. P. 16

[24] Стромыло Е.В., Барышников Г.В., Минаев Б.Ф., Григорас М. // Опт. и спектр. 2015. Т. 118. № 5. С. 735; Stromylo E.V., Baryshnikov G.V., Minaev B.F., Grigoras M. // Opt. Spectrosc. 2015. V. 118. N 5. P. 703.

[25] Барышников Г.В., Минаев Б.Ф., Минаева В.А., Слеnеu, A.A. // Опт. и спектр. 2014. Т. 116. № 3. С. 462; Baryshnikov G.V., Minaev B.F., Slepets A.A., Minaeva V.A. // Opt. Spectrosc. 2014. V. 116. N 3. P. 431.

[26] Барышников Г.В., Минаева В.А., Минаев Б.Ф., Сун В.-Х., Григорас М. // Опт. и спектр. 2016. Т. 121. № 3. С. 377; Baryshnikov G.V., Minaeva V.A., Minaev B.F., Sun V.-H., Grigoras M. // Opt. Spectrosc. 2016. V. 121. N 3. P. 348. 